\title{
Suplementación de testosterona durante el climaterio en mujeres con trastorno por deseo sexual hipoactivo
}

\author{
Testosterone supplementation during the climacteric period in women with hypoactive sexual desire disorder
}

Nicole Crimer ${ }^{a}$, Sergio Adrián Terrasa ${ }^{a, b, c}$ (D)

\begin{abstract}
Resumen
A partir del caso de una paciente con trastorno por deseo sexual hipoactivo durante su climaterio y a través del resumen de los resultados de dos revisiones sistemáticas, los autores de este artículo revisan la evidencia sobre la suplementación con andrógenos para el tratamiento de esta condición clínica. Concluyen que su uso sería relativamente seguro a corto plazo, aunque su eficacia no alcanzaría la relevancia clínica y no contamos aún con mayor información sobre la seguridad en el largo plazo. Los autores destacan además que el abordaje de las pacientes con este problema de salud debería ser realizado en forma integral, incluyendo opciones terapéuticas no farmacológicas e informando sobre las incertidumbres todavía presentes.

Abstract

Based on the case of a patient with hypoactive sexual desire disorder during her climacteric period and through the summary of the results of two systematic reviews, the authors of this article review the evidence supporting androgen supplementation for the treatment of this clinical condition. They conclude that its use would be relatively safe in the short term, although its efficacy would not reach clinical relevance and no further information on long-term safety is available. The authors also highlight that patients with this health problem should be approached comprehensively, including nonpharmacological therapeutic options and providing information on the uncertainties still present.
\end{abstract}

Palabras clave: Climaterio, Disfunciones Sexuales Femeninas, Deseo Sexual Hipoactivo, Testosterona. Keywords: Climateric, Female Sexual Dysfunctions, Hypoactive Sexual Desire, Testosterone.

Crimer N, Terrasa SA. Suplementación de testosterona durante el climaterio en mujeres con trastorno por deseo sexual hipoactivo. Evid Actual Pract Ambul. 2021;24(3):e002106. Available from: https://dx.doi.org/10.51987/EVIDENCIA.V24I4.6961.

\section{Caso clínico}

Se presenta al consultorio de su médica de cabecera una paciente de 52 años, en menopausia, sin antecedentes clínicos de relevancia, que no recibe medicación. Trae a la consulta la preocupación por la disminución de su deseo sexual. Refiere estar en pareja hace muchos años y notar que hace más de seis meses presenta falta de deseo sexual. Manifiesta que tiene muy buen vínculo con su pareja. Al interrogatorio dirigido, refiere escasos pensamientos eróticos y que esto es algo que le preocupa, ya que desea recuperar las ganas de practicar actividad sexual. Consulta a su médica porque leyó en una revista que una mujer famosa de otro país, también en etapa de posmenopausia, probó "los parches sexuales" y manifestó un resultado satisfactorio. Desea saber si ese tratamiento mejoraría su libido.

Tras realizar la anamnesis, la médica establece el diagnóstico de trastorno por deseo sexual hipoactivo y decide buscar la evidencia más actualizada sobre su tratamiento con parches transdérmicos de testosterona. Se aclara que éstos son diferentes al "chip" o "pellet" de testosterona, dispositivo que se implanta en el espacio subdérmico.

\section{Pregunta que generó el caso}

En pacientes climatéricas con deseo sexual hipoactivo, ¿los parches de testosterona aumentan la libido en comparación con el placebo?

- Paciente: mujeres en posmenopausia natural (no quirúrgica) con deseo sexual hipoactivo

- Intervención: parches de testosterona

- Comparación: placebo

- Desenlace: aumento del deseo sexual

\section{Estrategia de búsqueda}

Se realizó una búsqueda en PubMed incluyendo la combinación de términos female hypoactive sexual desire disorder AND testosterone. Se aplicaron los filtros de revisiones sistemáticas, meta-análisis y estudios publicados en los últimos 5 años. Se seleccionaron dos estudios que se describen a continuación.

\section{Algunos datos sobre el trastorno por deseo sexual hipoactivo}

El trastorno por el deseo sexual hipoactivo está definido por la ausencia o la reducción significativa del interés y/o la excitación sexual en al menos tres de los siguientes componentes: 1) interés en la actividad sexual, 2) pensamientos o fantasías sexuales, 3) iniciación de la actividad sexual, 4) excitación o placer sexual durante la actividad sexual en casi todos los encuentros, 5) en respuesta a cualquier señal erótica externa o interna, 6) de las sensaciones genitales o no genitales durante la actividad sexual en casi todos los encuentros sexuales ${ }^{1}$.

Durante el climaterio (etapa no reproductiva del ciclo sexual femenino) se producen cambios hormonales por el cese de la actividad folicular ovárica, con la consecuente hipoestrogene$\mathrm{mia}^{2}$. Si bien abordaje de las disfunciones sexuales debiera ser integral, y sus causas se clasifican en vinculares, biológicas y psicológicas, existe una probabilidad de alrededor del $60 \%$ de que, durante el climaterio, la causa del trastorno por el deseo sexual hipoactivo esté vinculada a la disminución de la síntesis hormonal $^{3}$.

Si bien los niveles de andrógenos endógenos no predicen la función sexual de las mujeres, existe evidencia de que la suplementación con una dosis de andrógenos que aumente sus con-

\footnotetext{
a Servicio de Medicina Familiar y Comunitaria, Hospital Italiano de Buenos Aires. nicole.crimer@hospitalitaliano.org.ar,

b Departamento de Investigación, Hospital Italiano de Buenos Aires.

c Departamento de Medicina, Instituto Universitario Hospital Italiano de Buenos Aires. sergio.terrasa@hospitalitaliano.org.ar
} 
centraciones séricas al límite superior de lo normal mejora la función sexual femenina en poblaciones seleccionadas de mujeres posmenopáusicas ${ }^{4,5}$. De todos modos, estos niveles no serían seguros, motivo por el cual están desaconsejados.

\section{Resumen de la evidencia}

Para contestar la pregunta que motivó el caso, fueron identificadas dos revisiones sistemáticas (RS). Una de ellas realizó una síntesis cualitativa de la evidencia ${ }^{6}$, y la otra, realizó el metaanálisis de siete ensayos clínicos ${ }^{7}$. La mayoría de los trabajos incluidos habían implementado un seguimiento de 24 semanas.

Como podemos observar en la Tabla 1, ambas RS documentaron diferencias estadísticamente significativas a favor del grupo tratado con testosterona, que no alcanzaron la mínima diferencia clínicamente relevante ${ }^{8}$.

Achilli C, et al. Efficacy and safety of transdermal testosterone in postmenopausal women with hypoactive sexual desire disorder: a systematic review and meta-analysis. Fertil Steril.

2017;107(2):475-482.e15

Objetivo. Revisar y sintetizar sistemáticamente la evidencia disponible en relación a la eficacia y seguridad de la testosterona transdérmica en mujeres posmenopáusicas para el tratamiento del trastorno por el deseo sexual hipoactivo.

Diseño. RS y meta-análisis.

Pacientes e intervenciones. Siete ensayos clínicos aleatorizados (ECA) enrolaron 3.035 participantes; 1.350 mujeres fueron aleatorizadas al tratamiento con el parche de testosterona, y 1.379 fueron aleatorizadas a recibir placebo.

Medidas de desenlace. Primario: episodios de satisfacción sexual. Secundarios: actividad sexual, orgasmo, dominio deseo de la herramienta Profile of Female Sexual Function, puntuación de angustia personal, eventos adversos, acné, aumento del crecimiento del cabello, vello facial, alopecia, cambios en la voz, síntomas urinarios, dolor mamario, cefalea, reacción en el sitio de aplicación, eventos adversos totales, eventos adversos graves, retiro del estudio y tasa de seguimiento.

Resultados. El grupo asignado a testosterona tuvo más episodios sexuales satisfactorios, actividad sexual, orgasmos, deseo, cambios significativos en la puntuación de la escala de angustia personal, eventos adversos androgénicos, acné y crecimiento del vello en comparación con el grupo placebo (ver Tabla 1). No hubo diferencias significativas entre los dos grupos en el aumento del vello facial, alopecia, cambios de la voz, síntomas urinarios, dolor mamario, cefaleas, reacción en el lugar del parche, eventos adversos totales, eventos adversos graves, motivos de retiro del estudio o el número de mujeres que completaron el estudio.

Conclusiones. La eficacia a corto plazo en cuanto a la mejora de la función sexual y la seguridad de la testosterona transdérmica en mujeres menopáusicas, natural y quirúrgicamente afectadas por trastorno por el deseo sexual hipoactivo, con o sin terapia hormonal con estrógenos / progestágenos resultó evidente en esta revisión sistemática. El uso de testosterona transdérmica se asoció con un aumento de los eventos adversos androgénicos como el acné, pero no se asoció con ningún evento adverso grave ${ }^{7}$.

\section{Ganesan K, et al. Transdermal Testosterone in Female} Hypoactive Sexual Desire Disorder: A Rapid Qualitative Systematic Review Using Grading of Recommendations Assessment, Development and Evaluation. Cureus. 2018;10(3):e2401

El objetivo de esta RS cualitativa fue evaluar los estudios publicados utilizando el marco GRADE para la calidad de la evidencia con respecto al uso de testosterona como terapia a corto y largo plazo para el trastorno por el deseo sexual hipoactivo. Los autores establecieron una puntuación GRADE de 4 (evidencia alta) como punto de corte para la medida de calidad de los estudios publicados que evalúan el uso de testosterona transdérmica en el trastorno por el deseo sexual hipoactivo. Losdesenlaces de interés fueron el número total de actividad sexual satisfactoria, el número de orgasmos, el deseo sexual y el nivel de angustia, mediante el uso de escalas. Se identificaron cinco ECA que cumplieron los criterios de inclusión. Los estudios seleccionados fueron de alta evidencia basada en la puntuación GRADE, ya que dos de los estudios obtuvieron 4 puntos, los otros dos estudios obtuvieron 5 puntos y un estudio obtuvo 6 puntos. Todos los estudios seleccionados de alta calidad tuvieron resultados similares, que sugieren una alta efectividad para el uso de $300 \mu \mathrm{g} / \mathrm{d}$ de testosterona transdérmica con o sin estrógeno para el manejo del trastorno por deseo sexual hipoactivo, con efectos secundarios mínimos. Un estudio mostró una tendencia a un mayor riesgo de cáncer de mama en el uso a largo plazo $(0,37 \%)$. Sin embargo, se necesitan más investigaciones prospectivas para evaluar los efectos secundarios más graves, como el cáncer de mama, en el uso prolongado de esta terapia ${ }^{6}$.

Tabla 1. Diferencias entre el grupo que recibió parches de testosterona respecto del grupo control en los tres principales resultados evaluados por ambas revisiones sistemáticas. Notas: *Rango. La mayoría de las diferencias fueron estadísticamente significativas. Solamente tres de los cinco trabajos incluidos en la revisión sistemática habían comunicado intervalos de confianza de sus estimadores. IC: intervalo de confianza; RR: riesgo relativo (razón de incidencias).

\begin{tabular}{|c|c|c|c|}
\hline Desenlaces & $\begin{array}{l}\text { Mínima diferencia } \\
\text { clinicamente relevante }^{8}\end{array}$ & $\begin{array}{l}\text { Achilli } 2017^{7} \\
\text { (7 ensayos) } \mathrm{N}=3.035\end{array}$ & $\begin{array}{l}\text { Ganesan } 2018^{6} \\
\text { (5 ensayos) } N=1.062\end{array}$ \\
\hline $\begin{array}{l}\text { Número de episodios sexuales satisfactorios duran- } \\
\text { te las últimas cuatro semanas, DM (IC 95\%) }\end{array}$ & 1 episodio & $+0,92(0,65$ a 1,19$)$ & $+1,2 \mathrm{a}+8,1^{*}$ \\
\hline $\begin{array}{l}\text { Índice de perfil de deseo sexual femenino (a mayor } \\
\text { puntaje, mayor bienestar), DM (IC 95\%) }\end{array}$ & 8 puntos & $+6,09(4,51$ a 7,68$)$ & $+5,3 \mathrm{a}+7,5^{\star}$ puntos \\
\hline $\begin{array}{l}\text { Puntaje personal de distrés (a menor puntaje, menor } \\
\text { distrés), DM (IC 95\%) }\end{array}$ & 20 puntos & $-8(-5,7$ a $-10,6)$ & $-9 a-11^{*}$ puntos \\
\hline $\begin{array}{l}\text { Incidencia de efectos adversos severos, RR (IC } \\
95 \%)\end{array}$ & No aplica & $1,01(0,97$ a 1,05$)$ & Sin diferencias \\
\hline $\begin{array}{l}\text { Diferencias en la incidencia de cáncer mamario a las } \\
52 \text { semanas (un solo estudio) }{ }^{9}\end{array}$ & No aplica & No comunicada & $+0,37 / 100(0,05$ a 1,35$)$ \\
\hline
\end{tabular}


Los resultados no fueron diferentes en el subgrupo de pacientes cuya menopausia había sido de origen quirúrgico. Desarrollaron acné un $11,97 \%$ de las pacientes que recibieron testosterona y un $7,71 \%$ de las que recibieron placebo (riesgo relativo [RR] 1,56; intervalo de confianza [IC] del $95 \% 1,17$ a 2,09).

\section{Conclusiones y recomendaciones}

A partir de la lectura de las dos revisiones sistemáticas resumidas $^{6,7}$, podemos concluir que, si bien el uso de testosterona en dosis de 300 microgramos por día para mujeres con trastorno del deseo sexual hipoactivo durante el climaterio podría tener algún rol en el tratamiento, la significancia clínica de sus beneficios es cuestionable. Por otro lado, un sólo estudio evaluó con un año de seguimiento la seguridad de esta intervención e informó la detección de al menos tres diagnósticos de cáncer de mama en el grupo que recibió el principio activo ${ }^{9}$. Son necesarios más estudios, con seguimiento a largo plazo, para que sean mejor evaluados los efectos adversos de esta terapia, que aún no ha sido aprobada por la Administración de Drogas y Alimentos de Estados Unidos (FDA, por sus iniciales en inglés) para el tratamiento de la disfunción sexual femenina. La escasez de investigaciones de buena calidad para determinar el balance de beneficios y riesgos de tratamientos farmacológicos para los problemas sexuales de la mujer podría interpretarse como una desventaja de género, ya que sí se cuenta con estudios más que suficientes sobre la seguridad del fármaco utilizado en el trastorno sexual más prevalente masculino -el sildenafil, para la disfunción eréctil-

Durante el año 2014, y en el contexto del Congreso Norteamericano de Obstetras y Ginecólogos, fue desarrollada la Guía de Práctica Clínica de recomendaciones para el uso de andrógenos en mujeres, con el aval de la Sociedad Norteamericana de Endocrinología y Medicina Reproductiva, la Sociedad Europea de Endocrinología y la Sociedad Internacional de Menopausia ${ }^{5}$. En sus conclusiones enfatizaron la importancia de no pesquisar la deficiencia de andrógenos en mujeres asintomáticas, ya que el significado de una eventual deficiencia no tendría una interpretación clara en ese escenario. Además, se expidieron en contra de la prescripción de testosterona en situaciones clínicas diferentes al deseo sexual hipoactivo.

Más recientemente, en mayo de 2021, se publicó una guía de práctica clínica para el uso de testosterona sistémica en el trastorno por el deseo sexual hipoactivo, desarrollada por la Sociedad Internacional para el estudio de la salud sexual de la mujer ${ }^{10}$ en base en los hallazgos de una revisión sistemática ${ }^{11}$ que incluyó estudios publicados y no publicados con formulaciones de testosterona para administración por diferentes vías, en mujeres pre y posmenopáusicas. En el meta-análisis realizado por Islam et al. ${ }^{11}$ de los estudios que compararon el uso de parches de testosterona vs. placebo, la diferencia de medias (DM) en el número de episodios sexuales satisfactorios fue de 0,85 (IC 95\% 0,52 a
1,18; 9 estudios; 3.238 mujeres con menopausia natural o quirúrgica), eficacia similar a la estimada por Achilli et al.\$ y que tampoco alcanza la diferencia mínima clínicamente relevante (ver Tabla 1). Esta guía sugiere realizar una evaluación biopsicosocial y un enfoque de tratamiento para el uso seguro y eficaz de testosterona que incluye pautas de medición, indicaciones, formulaciones, prescripción, dosificación, monitoreo y seguimiento. La guía hace énfasis en que, si bien no se conocen efectos adversos graves a corto plazo con el uso de la testosterona fisiológica, se desconoce por completo su seguridad en el largo plazo, por lo que podría utilizarse esta terapia sólo en pacientes con trastorno por el deseo sexual hipoactivo no relacionado con factores modificables o comorbilidades, como por ej. problemas en la relación de pareja o de la salud mental. Menciona la necesidad de que las pacientes firmen un consentimiento informado antes de iniciar el tratamiento con esta terapia farmacológica, la que se realiza con formulaciones aprobadas para su uso en varones, y apela a la toma de decisiones compartidas entre el profesiona de la salud y la paciente para tanto para la discusión de su uso fuera de las indicaciones autorizadas por las agencias regulatorias de medicamentos (off-label), así como sobre sus beneficios y riesgos potenciales. Además, la guía destaca que el tratamiento para el deseo sexual hipoactivo continúa siendo una dificultad no resuelta, dada la falta de estudios de buena calidad que aclaren los aspectos de seguridad a largo plazo ${ }^{10}$.

Volviendo a considerar el caso de la paciente que motivó esta búsqueda, a pesar de que la evidencia no es del todo concluyente y de que no se dispone de formulaciones de testosterona transdérmica aprobadas para su uso en mujeres, si luego de informarle la eficacia documentada en un contexto experimental, los riesgos de su implementación a corto plazo y la incertidumbre sobre sus efectos adversos a largo plazo de esta terapia, ella considerara optar por una terapia de suplementación androgénica, la médica de familia podría indicársela -si está lo suficientemente entrenada para esta indicación off-label, ya que se requiere una dosificación cuidadosa de formulaciones para uso masculino- o derivarla con un especialista para realizar el tratamiento.

Como reflexión final, es importante destacar que los cambios fisiológicos que ocurren durante el climaterio son parte del proceso vital, y nuestro rol como médicos de atención primaria es acompañar esos cambios desde una perspectiva integral de salud, haciendo énfasis en no patologizar las diferentes etapas de la vida, pero también teniendo en cuenta que frente al avance farmacológico y la difusión mediática de los mismos, debemos estar preparados para responder a las inquietudes y necesidades de nuestros pacientes.

Recibido el 21/07/2021 Aceptado el 23/08/2021 y Publicado el 30/09/2021.

\section{Agradecimientos}

A Analía Cerqueira y Valeria Vietto por sus aportes como revisoras de pares de este manuscrito.

\section{Referencias}

1. Guía de consulta de los criterios diagnósticos del $D S M-5^{\circledR}$ : Spanish Edition of the Desk Reference to the Diagnostic Criteria From DSM $-5^{\circledR}$. American Psychiatric Pub; 2014.

2. Servicio de Medicina Familiar y Comunitaria del Hospital Italiano de Buenos Aires. Medicina Familiar y Práctica Ambulatoria. Kopitowski K, Carrete P, Barani M, et al., editors. Panamericana; 2016.

3. Blümel M, Araya HM, Riquelme RO. Prevalencia de los trastornos de la sexualidad en mujeres climatéricas: Influencia de la menopausia y de la terapia de reemplazo hormonal. Rev méd Chile. 2002;130:1131-1138. Available from: http://dx.doi.org/10.4067/S0034-98872002001000007;https: //www.scielo.cl/scielo.php?script=sci_arttext\&pid=S0034-98872002001000007.

4. Shifren JL, Davis SR. Androgens in postmenopausal women: a review. Menopause. 2017;24(8):970-979. Available from: 10.1097/GME. 0000000000000903

5. Wierman ME, Arlt W, Basson R, et al. Androgen therapy in women: a reappraisal: an Endocrine Society clinical practice guideline. J Clin Endocrinol Metab. 2014;99(10):3489-3510. Available from: 10.1210/jc.2014-2260. 
6. Ganesan K, Habboush Y, Sultan S. Transdermal Testosterone in Female Hypoactive Sexual Desire Disorder: A Rapid Qualitative Systematic Review Using Grading of Recommendations Assessment, Development and Evaluation. Cureus . 2018;10(3):e2401. Available from: 10.7759/cureus.2401.

7. Achilli C, Pundir J, Ramanathan P, et al. Efficacy and safety of transdermal testosterone in postmenopausal women with hypoactive sexual desire disorder: a systematic review and meta-analysis. Fertil Steril. 2017;107(2):475-482.e15. Available from: 10.1016/j.fertnstert.2016.10.028.

8. Derogatis LR, Graziottin A, Bitzer J, et al. Clinically Relevant Changes in Sexual Desire, Satisfying Sexual Activity and Personal Distress as Measured by the Profile of Female Sexual Function, Sexual Activity Log, and Personal Distress Scale in Postmenopausal Women with Hypoactive Sexual Desire Disorder. J Sex Med. 2009;6(1):175-183. Available from: 10.1111/j.1743-6109.2008.01058.x.

9. Davis SR, Moreau M, Kroll R, et al. Testosterone for low libido in postmenopausal women not taking estrogen. N Engl J Med. 2008;359(19):20052017. Available from: 10.1056/NEJMoa0707302.

10. Parish SJ, Simon JA, Davis SR, et al. International Society for the Study of Women's Sexual Health Clinical Practice Guideline for the Use of Systemic Testosterone for Hypoactive Sexual Desire Disorder in Women. J Sex Med. 2021;18(5):849-867. Available from: https://doi.org/10.1016/j.jsxm.2020. 10.009

11. Islam RM, Bell RJ, Green S, et al. Safety and efficacy of testosterone for women: a systematic review and meta-analysis of randomised controlled trial data. Lancet Diabetes Endocrinol. 2019;7(10):754-766. Available from: 10.1016/S2213-8587(19)30189-5. 